\title{
Zeitschrift \\ für anorganische und allgemeine Chemie
}

Gegründet 1892

Redaktion

G. Fritz

Karlsruhe
W. Hanke

Berlin
R. Hoppe

Gießen

Wissenschaftlicher Beirat
M. Baudler, Köln
G. Becker, Stuttgart
J. D. Corbett, Ames/Iowa
K. Dehnicke, Marburg
H.-H. Emons, Berlin
D. Fenske, Karlsruhe
E. Fluck, Frankfurt/M.
N. N. Greenwood, Leeds

P. Hagenmuller, Bordeaux

E. Hoyer, Leipzig

K. Issleib, Halle/S.

H. Jacobs, Dortmund

M. Jansen, Bonn

Hk. Müller-Buschbaum, Kiel

H. Oppermann, Dresden
H. W. Roesky, Göttingen

H. G. v. Schnering, Stuttgart

K. Seppelt, Berlin

A. Simon, Stuttgart

K.-H. Thiele, Merseburg

E. Uhlig, Jena

K. Wieghardt, Bochum

W. Wieker, Berlin

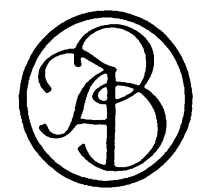

Johann Ambrosius Barth Leipzig - Heidelberg

Hüthig Verlagsgemeinschaft 


\title{
Zeitschrift für anorganische und allgemeine Chemie
}

\author{
Band 610 April 1992 S. $1-168$
}

\section{Inhalt}

E. Vielhaber, R. Hoppe (Gießen): Über Perrhenate. 3. Zum Aufbau des Mesoperrhenates $\mathrm{Na}_{3}\left[\mathrm{ReO}_{5}\right] \ldots \ldots$

B. Jung, G. Meyer (Hannover): Synthese, Kristallstruktur und thermischer Abbau von $\mathrm{Cs}_{1,5}\left[\mathrm{Re}_{3} \mathrm{I}_{3} \mathrm{Cl}_{7,5}\left(\mathrm{H}_{2} \mathrm{O}\right)_{1,5}\right]$

E. Manek, G. Meyer (Hannover): $\mathrm{Cs}_{4}\left[\mathrm{La}\left(\mathrm{NO}_{3}\right)_{6}\right]\left(\mathrm{NO}_{3}\right) \cdot \mathrm{HNO}_{3}$ : Das erste Salpetersäureaddukt eines ternären Alkali-Lanthanidnitrats

C. Michaelis, Hj. Mattausch, A. Simon (Stuttgart): $\mathrm{LnHal}_{2} \mathrm{H}_{\mathrm{n}}-$ Neue Phasen in den ternären Systemen

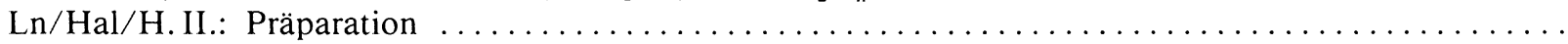

W. Müller, M. Jansen (Bonn): $\mathrm{A}_{4} \mathrm{~N}_{2} \mathrm{O}_{5}(\mathrm{~A}=\mathrm{Na}, \mathrm{K})$, neue Oxidnitrite der Alkalimetalle und eine Bemerkung zum quasi-binären System $\mathrm{K}_{3} \mathrm{NO}_{3} / \mathrm{K}_{3} \mathrm{OBr}$

G. Fritz, H. Rothmann, E. Matern (Karlsruhe): Die Phosphide $\mathrm{LiR}_{2} \mathrm{P}_{7}, \mathrm{Li}_{2} \mathrm{RP}_{7}(\mathrm{R}=\mathrm{Me} \mathrm{Si} \mathrm{Et}$, iPr, iBu) und

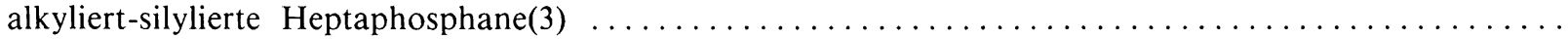

K. Brodersen, J. Hofmann (Erlangen): Quecksilberverbindungen mit Cyancarbanionen. II. Synthese und Kristallstruktur von Diquecksilber(I)-bis(1,1,3,3-tetracyanpropenid)

G. Fritz, B. Mayer (Karlsruhe): Komplexchemie P-reicher Phosphane und Silylphosphane. VII. Carbonylkomple-

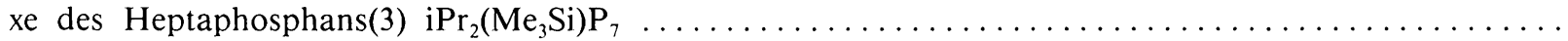

M. Lerch, W. Laqua (Gießen): Beiträge zu den Eigenschaften von Titanaten mit Ilmenitstruktur. II. Zur Thermodynamik und elektrischen Leitfähigkeit von $\mathrm{NiTiO}_{3}$ und anderen oxidischen Phasen mit Ilmenitstruktur

Th. Bremm, M. Jansen (Bonn): Verfeinerung der Kristallstruktur von $\mathrm{K}_{2} \mathrm{O}_{2}$

N. Ruchaud, J. Grannec, P. Gravereau, P. Nuñez, A. Tressaud, W. Massa, G. Frenzen, D. Babel (Talence/France, Marburg): Copper Weberites: Crystal Structure and Magnetic Investigation of $\mathrm{Na}_{2} \mathrm{CuGaF}_{7}$ and $\mathrm{Na}_{2} \mathrm{CuInF}_{7}$

P. Jutzi, S. Opiela (Bielefeld): Synthese und Eigenschaften Pentamethylcyclopentadienyl-substituierter PPC- bzw. AsPC-Dreiring-Systeme

J. Bill, R. Riedel, G. Passing (Stuttgart): Amin-Borane als Precusoren für Borcarbidnitrid

G. Demazeau, S. H. Byeon, J. H. Choy, P. Hagenmuller (Talence/France, Seoul/Korea): Influence des Liaisons Chimiques sur la Transition Spin Faible $\rightarrow$ Spin Fort du Cobalt(III) $\left(3 d^{6}\right)$ au sein D'Oxydes de Type $\mathrm{K}_{2} \mathrm{NiF}_{4}$

M. Jansen, S. Rings, H. P. Baldus (Bonn): Darstellung und Kristallstruktur von Tetrakis(pentafluorphenylamino)silan 
C. Schneidersmann, R. Hoppe (Gießen): Neue Alkalioxoarsenate(V). Zur Kenntnis von $\mathrm{Rb}_{2} \mathrm{Li}\left[\mathrm{AsO} \mathrm{O}_{4}\right]$ und

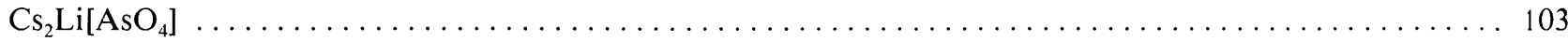

St. Vogler, W. Massa, K. Dehnicke (Marburg): [Na(15-Krone-5)][ReFCl $\left.(\mathrm{NO})\left(\mathrm{CH}_{3} \mathrm{CN}\right)\right]$ - Synthese, IR-Spek-

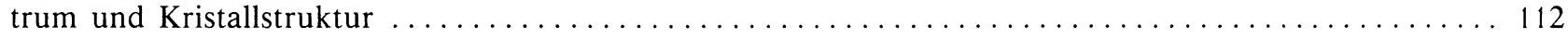

I. Sens, H. Stenger, U. Müller, K. Dehnicke (Marburg): Die Kristallstruktur von $\left[\mathrm{MoO}_{2} \mathrm{~F}_{2}\left(2,2^{\prime}\right.\right.$-Bipyridyl)] $\ldots \ldots 117$

W. Schnick, J. Lücke (Bonn): Darstellung, Kristallstruktur und IR-spektroskopische Untersuchung von Phos-

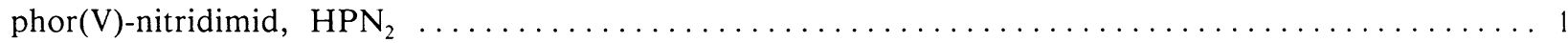

A. Zschunke, C. Mügge, M. v. Löwis, F. Krech, L. Weber, H. Schumann, H. G. Stammler (Berlin, Halle/S., Bielefeld): $\gamma$-anti-Effekt an 1-Phosphabicyclo[3.3.1]nonan-Derivaten mit Substituenten am Phosphor. Synthese und Struktur von Pentacarbonyl(1-phosphabicyclo[3.3.1]nonan)chrom $\ldots \ldots \ldots \ldots \ldots \ldots \ldots \ldots 127$

G.-Q. Wu, Z. Tang, Y. Yu, P. Lin, M. Jansen, K. Königstein (Bejing/China, Bonn): A New Type of Divalent Europium Compound $\mathrm{MEuPO}_{4}(\mathrm{M}=\mathrm{K}, \mathrm{Rb}, \mathrm{Cs})$, its Synthesis, Crystal Structure, and Properties ........ 135

M. Jansen, B. Begemann, J. Geb (Bonn): Mischkristallbildung in den Systemen $\mathrm{As}_{2} \mathrm{O}_{5} / \mathrm{SbAsO}_{5}$ und $\mathrm{As}_{2} \mathrm{O}_{5} / \mathrm{AsPO}_{5}$. Bestimmung der spontanen Deformationen und Verfeinerung der Kristallstrukturen der ter-

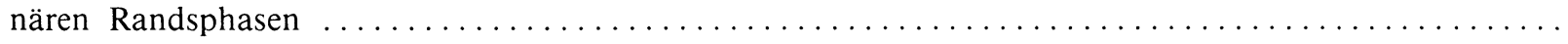

K. Hensen, H. Rossmann, W. Bensch (Frankfurt/M.): Die Kristallstrukturen von $\mathrm{Me}_{3} \mathrm{SiI} \cdot \beta$-Picolin und $\mathrm{Me}_{3} \mathrm{SiI} \cdot \gamma$-Picolin. Vergleich der Lewis-Basen Pyridin, $\beta$-Picolin und $\gamma$-Picolin $\ldots \ldots \ldots \ldots \ldots \ldots \ldots 145$

B. Bovio, A. Cingolani, F. Bonati (Pavia/Italy, Camerino/Italy): Bis(pyrazol-1-yl)propane as a bulky Ligand: Xray Crystal Structure Determination of its Adduct with Zinc(II) Chloride

Y. Soneda, M. Inagaki (Sapporo/Japan): Conditions for the Formation of a New Type of Graphite Intercalation

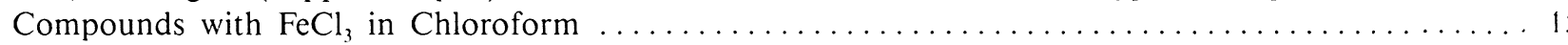

D. Labahn, S. Brooker, G. M. Sheldrick, H. W. Roesky (Göttingen): Synthese und Kristallstrukturen und m.onomeren Bis(thiophenolato)metall(II)-Komplexen 


\title{
Darstellung, Kristallstruktur und IR-spektroskopische Untersuchung von Phosphor(V)-nitrid-imid, $\mathrm{HPN}_{2}$
}

\author{
W. Schnick* und J. Lücke \\ Bonn, Insti:ut für Anorganische Chemie der Universität
}

Bei der Recaktion eingegangen am 25. August 1991.

Inhaltsuibersicht. Reines und feinkristallines Phosphor(V)-n.trid-imid $\left(\mathrm{HPN}_{2}\right)$ wurde durch heterogene Ammonol/se von $\mathrm{P}_{3} \mathrm{~N}_{5}$ mit gasförmigem $\mathrm{NH}_{3}(\mathrm{~T}=$ $580^{\circ} \mathrm{C}, \mathrm{p}=30$ bar, $6 \mathrm{~d}$ ) dargestellt. Die Kristallstruktur von HPN. wurde auf der Basis von Röntgen-Pulverdiffraktometzrdaten mit Hilfe der Rietveld-Methode verfei-

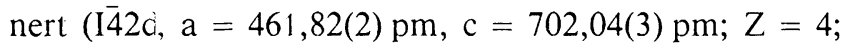
41 beobashtete Reflexe, $17^{\circ}<2 \Theta<125^{\circ} ; \mathrm{Cu}-\mathrm{K} \alpha_{1}$,
Germanium-Monochromator; $\mathrm{R}(\mathrm{wp})=0,072 ; \mathrm{R}(\mathrm{I}, \mathrm{hkl})$ $=0,048)$. Im Festkörper ist HPN 2 aus einem dreidimensionalen Netz allseitig eckenverknüpfter $\mathrm{PN}_{4}$-Tetraeder $\left(\mathrm{P}-\mathrm{N}: 159,9(4) \mathrm{pm} ; \mathrm{P}-\mathrm{N}-\mathrm{P}: 130,1(4)^{\circ}\right)$ aufgebaut. Die $\mathrm{H}$-Atome sind kovalent an die Hälfte der N-Atome gebunden. Im IR-Spektrum werden sechs Schwingungsbanden $\left(v(\mathrm{~N}-\mathrm{H}): 3224 ; v_{\mathrm{as}}(\mathrm{PNP}): 1330,1223 ; v_{\text {as }}(\mathrm{PNHP})\right.$ : 971, $\left.901 ; \delta(\mathrm{PNP}): 531 \mathrm{~cm}^{-1}\right)$ beobachtet.

\section{Preparation, Crystal Structure, and IR-spectroscopic Investigation of Phosphorus Nitride Imide, $\mathrm{HPN}_{2}$}

\begin{abstract}
Pure and fine crystalline phosphorus nitride imide $\left(\mathrm{H}^{?} \mathrm{~N}_{2}\right)$ is obtained by heterogeneous ammonolysis of $\mathrm{P}_{3} \mathrm{Il}_{5}$ with gaseous $\mathrm{NH}_{3}\left(T=580{ }^{\circ} \mathrm{C}, \mathrm{p}=30 \mathrm{bar}\right.$, $6 \mathrm{~d})$. X-ray powder diffraction data has been used to refine the crystal structure of $\mathrm{HPN}_{2}$ by the Rietveld fullprofile technique $(\bar{I} \overline{2} \mathrm{~d} ; \quad \mathrm{a}=461.82(2) \mathrm{pm}, \quad \mathrm{c}=$ $702.04(3) \mathrm{pm} ; \quad \mathrm{Z}=4 ; \quad 41$ reflections observed, $17^{\circ}<2 \Theta<125^{\circ}$, CuK $\alpha_{1}$, germanium monochromator; $\mathrm{R}(w p)=0.072, \mathrm{R}(\mathrm{I}, \mathrm{hkl})=0.048)$. In the solid $\mathrm{HPN}_{2}$
\end{abstract}

contains a three-dimensional framework of corner-sharing $\mathrm{PN}_{4}$-tetrahedra $(\mathrm{P}-\mathrm{N}: 159.9(4) \mathrm{pm} ; \mathrm{P}-\mathrm{N}-\mathrm{P}$ : $130.1(4)^{\circ}$. The hydrogen atoms are covalently bonded to half of the nitrogen atoms. The IR spectrum exhibits six vibrational modes $\left(v(\mathrm{~N}-\mathrm{H}): 3224 ; \quad v_{\text {as }}(\mathrm{PNP}): 1330\right.$, $\left.1223 ; v_{\text {as }}(\mathrm{PNHP}): 971,901 ; \delta(\mathrm{PNP}): 531 \mathrm{~cm}^{-1}\right)$.

Key words: Phosphorus nitride imide; preparation; crystal structure; i. r. data

\section{Einleiturg}

Gemeinsımes strukturelles Bauelement von Phosphor(V) nitriden sind $\mathrm{PN}_{4}$-Tetraeder, die unterschiedlich über geneinsame Ecken miteinander verknüpft sein können. Von , isolierten" Ortho-Anion $\mathrm{PN}_{4}{ }^{7-}$ in $\mathrm{Li}_{7} \mathrm{PN}_{4}[1]$ bis zu einer dem $\beta$-Cristobalit isosteren $\mathrm{P}-\mathrm{N}$-Teilstruktur ${ }_{\infty}^{3}\left[\mathrm{PN}_{4 / 2}{ }^{-}\right]$in $\mathrm{LiPN}_{2}$ [2] werden dabei ganz verschiedene Konden:ationsgrade beobachtet: In $\mathrm{Li}_{12} \mathrm{P}_{3} \mathrm{~N}_{9}$ erhält man Cyclot rishosphat-analoge $\mathrm{P}_{3} \mathrm{~N}_{9}{ }^{12-}$-Ringe [3], das Nitrido-Anabgon zu molekularem Phosphor(V)-oxid wurde als $\mathrm{P}_{4} \mathrm{~N}_{11}{ }^{10-}$-Anion in $\mathrm{Li}_{10} \mathrm{P}_{4} \mathrm{~N}_{10}$ entdeckt [4]. Als weiteres
Beispiel einer dreidimensional unendlich verknüpften $\mathrm{P}$-N-Teilstruktur fanden wir kürzlich $\mathrm{Zn}_{7}\left[\mathrm{P}_{12} \mathrm{~N}_{24}\right] \mathrm{Cl}_{2}$, einen Sodalith mit einem Phosphor-Stickstoff-Grundgerüst [5].

Im Gegensatz zu diesen gut charakterisierten MetallPhosphor(V)-nitriden lagen bislang nur unzureichende und widersprüchliche Erkenntnisse über Darstellung, Eigenschaften und Strukturen von wasserstoffhaltigen Phosphor(V)-nitriden vor. Obwohl bereits 1846 eine Verbindung der empirischen Zusammensetzung $\mathrm{HPN}_{2}$ erwähnt wurde, die später die Bezeichnung „,Phospham“ erhielt [6-8], ist es in der Vergangenheit nicht gelungen, 
$\mathrm{HPN}_{2}$ in reiner, definierter und kristalliner Form darzustellen. Vielmehr entstanden bei früheren Arbeiten oft undefinierte Mischungen verschiedener oligomerer und polymerer $\mathrm{H}-\mathrm{P}-\mathrm{N}$-Phasen, welche in röntgenamorphem Zustand anfielen und sich nicht weiter charakterisieren ließen [9-12]. Miller und Mitarb. berichteten zwar über kristallines $\mathrm{HPN}_{2}$ und vermuteten strukturelle Analogie zu LiPN 2 [13], offensichtlich die gleiche Substanz wurde jedoch von anderen Autoren als $\gamma-\mathrm{P}_{3} \mathrm{~N}_{5}$ bezeichnet [14]. Da bislang weder eine verläßliche Darstellungsmethode noch genaue strukturelle Daten für kristallines $\mathrm{HPN}_{2}$ mitgeteilt wurden, haben wir uns im Rahmen systematischer Untersuchungen binärer und ternärer Phosphor(V)-nitride [1-5] mit der Reindarstellung und Strukturuntersuchung von Phosphor(V)-nitrid-imid $\mathrm{HPN}_{2}$ beschäftigt, worüber wir in dieser Arbeit berichten.

\section{Darstellung und Eigenschaften}

Obwohl wasserstoffhaltige $\mathrm{P}-\mathrm{N}$-Verbindungen wechselnder Zusammensetzung bei der Darstellung von $\mathrm{P}_{3} \mathrm{~N}_{5}$ durch heterogene Umsetzung von gasförmigem Ammoniak mit Hexachlorcyclotriphosphazen $\left(\left(\mathrm{PNCl}_{2}\right)_{3}\right)$ als Zwischen- und Nebenprodukte auftreten, gelingt es nicht, die Ammonolyse und Kondensation der oligomeren bzw. polymeren Produkte auf der Stufe definierter, einphasiger wasserstoffhaltiger Phosphor(V)-nitride anzuhalten. Aus diesem Grund erschien uns die Ammonolyse von reinem $\mathrm{P}_{3} \mathrm{~N}_{5}$ für die Darstellung von $\mathrm{HPN}_{2}$ am besten geeignet zu sein.

\section{1 $P_{3} N_{5}$}

Phosphor(V)-nitrid $\mathrm{P}_{3} \mathrm{~N}_{5}$ [15] wird durch Umsetzung stöchiometrischer Mengen $\mathrm{NH}_{4} \mathrm{Cl}$ (p. A., Riedel-de Haen, Seelze) und $\left(\mathrm{PNCl}_{2}\right)_{3}$ (p. A., Merck-Schuchardt) in dickwandigen, druckstabilen Quarzampullen (Innendurchmesser: $10 \mathrm{~mm}$, Länge: $150 \mathrm{~mm}$ ) bei $780^{\circ} \mathrm{C}$ (Reaktionsdauer: $2 \mathrm{~d}$, Ansatz: $1 / 2 \mathrm{mmol}$ ) dargestellt (Gl. (1)).

$2 \mathrm{NH}_{4} \mathrm{Cl}+\left(\mathrm{PNCl}_{2}\right)_{3} \underset{2 \mathrm{~d}}{\stackrel{780^{\circ} \mathrm{C}}{\longrightarrow}} \mathrm{P}_{3} \mathrm{~N}_{5}+8 \mathrm{HCl}$

Das entstehende Produkt ist farblos und mikrokristallin (Kristallitgröße unter dem Rasterelektronenmikroskop $\leqq 10 \mu \mathrm{m})$. Phosphor(V)-nitrid ist luftstabil und unter

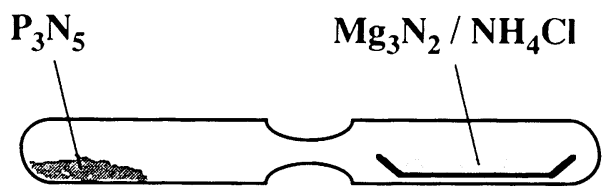

T

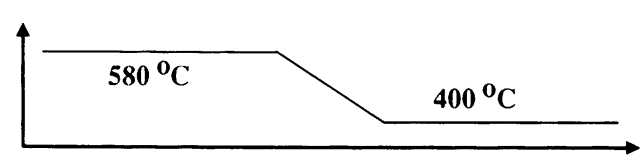

Abb. 1 Gefüllte Quarzampulle im Temperaturgradienten zur Darstellung von $\mathrm{HPN}_{2}$ durch Druck-Ammonolyse von $\mathrm{P}_{3} \mathrm{~N}_{5}$
Normalbedingungen unlöslich in Säuren, Basen und allen gängigen Lösemitteln. Die thermische Zersetzung von $\mathrm{P}_{3} \mathrm{~N}_{5}$ im Vakuum bzw. unter Argonatmosphäre setzt oberhalb von etwa $900{ }^{\circ} \mathrm{C}$ unter $\mathrm{N}_{2}$-Abspaltung ein, wobei röntgenamorphe und undefinierte braune Produkte entstehen. In der DTA/TG-Untersuchung wird eine allmähliche Dissoziation beobachtet, ein definierter Zersetzungspunkt tritt nicht auf.

Zur Elementaranalyse wurden die erhaltenen Proben in einem speziellen Aufschlußsystem [16] bei $190^{\circ} \mathrm{C}$ und 12 bar (Reaktionsdauer: 2d) in verd. Schwefelsäure gelöst, wobei Phosphor(V)-nitrid zu Ammonium-hydrogen-phosphat hydrolysiert. Phosphor wurde photometrisch als Molybdo-vanadato-phosphat, Stickstoff als Indophenol bestimmt. Chlor oder Silicium konnten weder im Festkörper (EDX-Analyse) noch in den gelösten Proben nachgewiesen werden. Sauerstoff ließ sich im Festkörper mittels WDX-Analyse ebenfalls nicht nachweisen (Nachweisgrenze: ca. 0,5 Gew.-\%). Die Abwesenheit von Wasserstoff $(\mathrm{N}-\mathrm{H})$ wurde IR-spektroskopisch überprüft.

\section{$1.2 \mathrm{HPN}_{2}$}

Die heterogene Ammonolyse (Gl. (2)) von $\mathrm{P}_{3} \mathrm{~N}_{5}$ wurde in dickwandigen Quarzampullen durchgeführt (Innendurchmesser: $10 \mathrm{~mm}$, Länge: $200 \mathrm{~mm}$; Ansatz: $1,5 \mathrm{mmol}$ $\mathrm{P}_{3} \mathrm{~N}_{5}$ ). Das benötigte Ammoniak (3facher Überschuß) wurde direkt in der Glasampulle durch räumlich getrennte Umsetzung von $\mathrm{NH}_{4} \mathrm{Cl}$ und $\mathrm{Mg}_{3} \mathrm{~N}_{2}$ (röntgenrein, dargestellt aus Magnesium-Spänen und $\mathrm{NH}_{3}, \quad 900^{\circ} \mathrm{C}$; $\mathrm{Mg} 99,9 \%$, Ventron; $\mathrm{NH}_{3} 99,8 \%$, BASF) erzeugt (Gl. (3)).

$\mathrm{P}_{3} \mathrm{~N}_{5}+\mathrm{NH}_{3} \frac{580^{\circ} \mathrm{C}}{14 \mathrm{~d}} \longrightarrow 3 \mathrm{HPN}_{2}$

$\mathrm{Mg}_{3} \mathrm{~N}_{2}+6 \mathrm{NH}_{4} \mathrm{Cl} \stackrel{400^{\circ} \mathrm{C}}{\longrightarrow} 8 \mathrm{NH}_{3}+3 \mathrm{MgCl}_{2}$

Zur Durchführung der Reaktion wurde die in Abb. 1 dargestellte Quarzampulle in einen horizontal angebrachten Zwei-Zonen-Röhrenofen $\left(580^{\circ} \mathrm{C} / 400^{\circ} \mathrm{C}\right)$ überführt. Phosphor(V)-nitrid-imid fällt bei dieser Darstellung in Form eines farblosen, feinkristallinen Pulvers an. Die Substanz ist luftstabil und unter Normalbedingungen unlöslich in Säuren, Basen und allen gängigen Lösemitteln. Nach kombinierten DTA/TG-Untersuchungen ist $\mathrm{HPN}_{2}$ im Vakuum bis $570^{\circ} \mathrm{C}$ thermisch stabil. Oberhalb dieser Temperatur tritt allmähliche endotherme Zersetzung unter Ammoniak-Abspaltung und Bildung von röntgenamorphem Phosphor(V)-nitrid $\left(\mathrm{P}_{3} \mathrm{~N}_{5}\right)$ ein.

Das erhaltene Produkt $\left(\mathrm{HPN}_{2}\right)$ wurde ebenfalls, wie für $\mathrm{P}_{3} \mathrm{~N}_{5}$ beschrieben, analysiert. Dabei konnten weder Silicium- noch Sauerstoffspuren im Festkörper nachgewiesen werden (WDX- und EDX-Analysen).

\section{Röntgenographische Untersuchungen}

Die Strukturverfeinerung von $\mathrm{HPN}_{2}$ (vgl. Tab. 1) wurde auf der Basis von Röntgen-Pulverdiffraktometer-Daten durchgeführt. Die Messungen erfolgten auf einem fokussierenden Transmissions-Pulverdiffraktometer STADI/P [17] unter Verwendung 
monochromatischer $\mathrm{CuK} \alpha_{1}$-Strahlung. Die untersuchten Pulverproben wurden in verschlossenen Glaskapillaren $(0,3 \mathrm{~mm})$ in Debye-Scherrer-Geometrie vermessen. Die Beugungsintensitäten wurden unter Verwendung eines linearen ortsempfindlichen Proportionaldetektors (MINI-PSD, Fa. Stoe [17], Öffnungswinkel: $6,7^{\circ}$ ) aufgezeichnet.

Tabelle $1 \mathrm{HPN}_{2}$, kristallographische Daten

\begin{tabular}{|c|c|}
\hline Kristallsystem & tetragonal \\
\hline Raumgruppe & $\mathrm{I} \overline{4} 2 \mathrm{~d}-\mathrm{D}_{2 \mathrm{~d}}^{12}(\mathrm{Nr} .122)$ \\
\hline Gitterkonstanten & $\begin{array}{l}\mathrm{a}=461,82(2) \mathrm{pm} \\
\mathrm{c}=702,04(3) \mathrm{pm}\end{array}$ \\
\hline $\begin{array}{l}\text { Volumen der Elementar- } \\
\text { zelle }\end{array}$ & $1,497 \cdot 10^{8} \mathrm{pm}^{3}$ \\
\hline $\begin{array}{l}\text { Anzahl Formeleinheiten } \\
\text { pro EZ. }\end{array}$ & $Z=4$ \\
\hline Strahlung, Monochromator & $\begin{array}{l}\text { CuK } \alpha_{1} \text {, Germanium } \\
(\lambda=154,056 \mathrm{pm})\end{array}$ \\
\hline Dichte (röntgenographisch) & $\rho=2,6 \mathrm{~g} \cdot \mathrm{cm}^{3}$ \\
\hline lin. Absorptionskoeffizient & $\mu=108,9 \mathrm{~cm}^{-2}$ \\
\hline \multirow[t]{2}{*}{ Diffraktometer/Detektor } & STOE STADI/P \\
\hline & $\begin{array}{l}\text { Transmissions-Pulverdiffrakto- } \\
\text { meter lin. ortsempfindlicher } \\
\text { Proportionalzähler (STOE- } \\
\text { MINI-PSD) }\end{array}$ \\
\hline Beugungswinkelbereich & $17^{\circ}<2 \Theta<125^{\circ}$ \\
\hline Anzahl Datenpunkte & 5401 \\
\hline Gesamtmeßzeit & $12 \mathrm{~h}$ \\
\hline Anzahl beob. Reflexe & 41 \\
\hline Anzahl Profilparameter & 17 \\
\hline Profilfunktion & Mod. 2 Lorentz-Funktion \\
\hline Anzahl verf. Ortsparameter & 1 \\
\hline $\begin{array}{l}\text { Anzahl verf. Temperatur- } \\
\text { faktoren }\end{array}$ & 2 \\
\hline$R(w p)$ (Profil) & 0,072 \\
\hline $\mathrm{R}(\mathrm{I}, \mathrm{hkl})$ (Struktur) & 0,048 \\
\hline
\end{tabular}

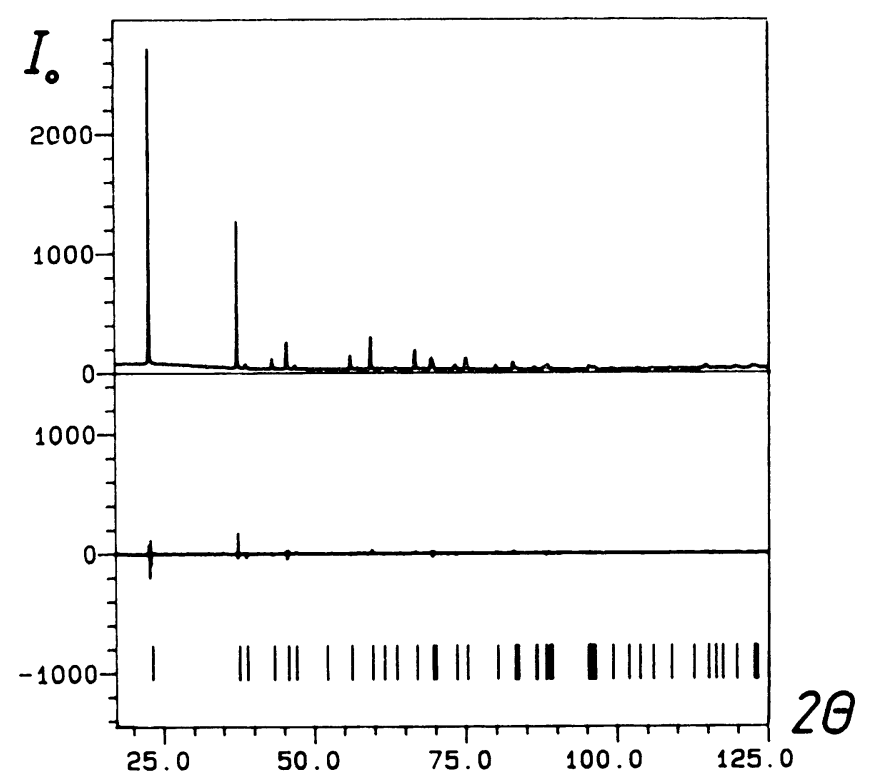

Abb. 2 Beobachtetes (oben) und Differenz-Röntgenpulverdiagramm $\left(\mathrm{I}_{\text {beob }}-\mathrm{I}_{\text {calc }}\right)$ sowie theoretische Lage der symmetrieerlaubten Beugungsreflexe von $\mathrm{HPN}_{2}$
Tabelle 2 Beobachtete und berechnete Röntgenpulverintensitäten $\mathrm{HPN}_{2}\left(\mathrm{CuK} \alpha_{1}\right.$-Strahlung)

\begin{tabular}{|c|c|c|c|c|}
\hline h k 1 & $2 \Theta_{\text {beob. }}\left[{ }^{\circ}\right]$ & FWHM [7] & $I_{\text {beob. }}$ & $I_{\text {theor. }}$ \\
\hline 101 & 23,03 & 0,14 & 2884,7 & 2871,6 \\
\hline 112 & 37,59 & 0,14 & 1129,1 & 1099,2 \\
\hline 200 & 38,97 & 0,14 & 51,5 & 66,2 \\
\hline 103 & 43,32 & 0,15 & 92,3 & 107,6 \\
\hline 211 & 45,76 & 0,15 & 302,5 & 287,0 \\
\hline 202 & 47,07 & 0,16 & 51,2 & 32,1 \\
\hline $\begin{array}{lll}0 & 0 & 4\end{array}$ & 52,08 & 0,17 & 0,0 & 3,8 \\
\hline 220 & 56,30 & 0,18 & 139,1 & 146,5 \\
\hline 213 & 59,67 & 0,20 & 336,9 & 316,5 \\
\hline 301 & 61,63 & 0,20 & 23,1 & 26,2 \\
\hline 310 & 63,67 & 0,21 & 25,2 & 21,9 \\
\hline 204 & 66,92 & 0,23 & 260,1 & 241,5 \\
\hline 312 & 69,68 & 0,25 & 216,5 & 218,9 \\
\hline 105 & 69,99 & 0,25 & 29,4 & 29,4 \\
\hline $\begin{array}{lll}3 & 0 & 3\end{array}$ & 73,60 & 0,27 & 90,7 & 87,9 \\
\hline 321 & 75,37 & 0,28 & 184,1 & 176,7 \\
\hline 224 & 80,25 & 0,32 & 58,5 & 52,2 \\
\hline 215 & 83,13 & 0,34 & 128,5 & 111,3 \\
\hline 400 & 83,70 & 0,35 & 31,3 & 35,5 \\
\hline 323 & 86,57 & 0,37 & 38,7 & 33,7 \\
\hline 314 & 86,67 & 0,37 & 14,9 & 13,1 \\
\hline $\begin{array}{lll}4 & 1 & 1\end{array}$ & 88,28 & 0,39 & 87,1 & 90,5 \\
\hline 116 & 88,77 & 0,39 & 84,6 & 68,9 \\
\hline 402 & 89,23 & 0,39 & 15,2 & 15,1 \\
\hline 206 & 95,15 & 0,45 & 3,6 & 2,0 \\
\hline 332 & 95,62 & 0,46 & 59,0 & 52,5 \\
\hline 305 & 95,91 & 0,46 & 45,7 & 45,2 \\
\hline 420 & 96,48 & 0,46 & 69,1 & 67,0 \\
\hline 413 & 99,36 & 0,49 & 26,9 & 30,7 \\
\hline 422 & 102,07 & 0,52 & 4,7 & 8,1 \\
\hline 107 & 103,65 & 0,54 & 22,6 & 34,0 \\
\hline 404 & 106,00 & 0,57 & 34,0 & 41,8 \\
\hline 325 & 109,00 & 0,61 & 40,5 & 25,1 \\
\hline 431 & 114,53 & 0,68 & 22,0 & 21,3 \\
\hline 501 & 114,53 & 0,68 & 20,9 & 20,2 \\
\hline 316 & 115,06 & 0,69 & 89,1 & 89,1 \\
\hline 510 & 116,53 & 0,71 & 13,0 & 9,2 \\
\hline 217 & 117,30 & 0,72 & 29,2 & 32,5 \\
\hline 424 & 119,88 & 0,75 & 52,8 & 64,4 \\
\hline $\begin{array}{lll}0 & 0 & 8\end{array}$ & 122,80 & 0,79 & 27,8 & 25,8 \\
\hline 512 & 122,89 & 0,80 & 95,6 & 91,2 \\
\hline 415 & 123,24 & 0,80 & 32,0 & 29,7 \\
\hline
\end{tabular}

Das Röntgenbeugungsdiagramm von $\mathrm{HPN}_{2}$ (Abb. 2) hat eine auffallende Ähnlichkeit mit dem von $\mathrm{LiPN}_{2}$ [2] und läßt sich ebenfalls tetragonal indizieren (vgl. Tab. 1)

Für die Strukturverfeinerung nach dem Rietveld-Verfahren [17] wurden Meßdaten (Tab. 2) im Beugungswinkelbereich von $17^{\circ}<2 \Theta<125^{\circ}$ (erster beobachteter Reflex bei $23,03^{\circ}$ ) herangezogen, welche zuvor durch einen Standard (Silicium) bezüglich der $2 \Theta$-Skala linear korrigert worden waren. Die Winkelabhängigkeit der Reflexhalbwertsbreiten (FWHM) sowie des Untergrundes konnte durch geeignete Tchebychev-Polynome beschrieben und mitverfeinert werden. Für alle beobachteten Reflexe wurde ein Integrationsbereich von jeweils 2,5 Halbwertsbreiten auf jener Seite der Reflexposition berücksichtigt. 
Die Profilparameter wurden vor Freigabe der Strukturparameter verfeinert. Als Startparameter für die Strukturverfeinerung dienten die für LiPN $\mathrm{N}_{2}$ bestimmten Ortsparameter für Phosphor und Stickstoff [2]. Die isotropen thermischen Schwingungsparameter wurden nach der Strukturverfeinerung unter Fixierung der Ortsparameter verfeinert. Die Rechnungen konvergierten gegen $R(w p)=0,072$ für das Profil und $R(I, h k l)=0,048$ für die Struktur. In einer sich anschließenden Differenz-Fourier-Synthese mit $\left[\mathrm{F}_{\mathrm{o}}-\mathrm{F}_{\mathrm{c}}\left(\mathrm{PN}_{2}{ }^{-}\right)\right]$als Fourierkoeffizienten wurden keine signifikanten Maxima mehr ermittelt.

Tabelle 3 Verfeinerte Ortsparameter und,,isotrope“ Temperaturfaktoren ${ }^{\text {a) }}$ mit Standardabweichungen in Einheiten der letzten Dezimalstelle

\begin{tabular}{llllll}
\hline Atom & $\begin{array}{l}\text { Wyckoff- } \\
\text { Symbol }\end{array}$ & $\mathrm{x} / \mathrm{a}$ & $\mathrm{y} / \mathrm{b}$ & $\mathrm{z} / \mathrm{c}$ & $\mathrm{U}($ iso $)$ \\
\hline $\mathrm{P}$ & $4 \mathrm{a}$ & 0 & 0 & 0 & $0,008(1)$ \\
$\mathrm{N}$ & $8 \mathrm{~d}$ & $0,1462(21)$ & $1 / 4$ & $1 / 8$ & $0,012(8)$ \\
$\mathrm{H}$ & $\left.8 \mathrm{~d}^{\mathrm{b}}\right)$ & 0,358 & $1 / 4$ & $1 / 8$ & - \\
\hline
\end{tabular}

a) Temperaturfaktorexponent in der Form $-8 \pi^{2} U_{\text {iso }} \sin ^{2} \theta / \lambda^{2}$

b) theoretisch berechnete H-Position (nicht verfeinert, $\mathrm{H}-\mathrm{N}$ : $98 \mathrm{pm}$ ), Lage halbbesetzt

Tabelle 4 Interatomare Abstände [pm] und Winkel $\left[^{\circ}\right]$ in $\mathrm{HPN}_{2}$

\begin{tabular}{lcl}
\hline $\mathrm{P}-\mathrm{N}$ & $159,9(4)$ & $(4 \mathrm{mal})$ \\
$\mathrm{N}-\mathrm{H}$ & 98 & (H-Position nicht verfeinert) \\
$\mathrm{P}-\mathrm{N}-\mathrm{P}$ & $130,1(4)$ & \\
$\mathrm{N}-\mathrm{P}-\mathrm{N}$ & $107,5(5)$ & $(4 \mathrm{mal})$ \\
& $113,4(5)$ & $(2 \mathrm{mal})$ \\
\hline
\end{tabular}

Die endgültigen Atomparameter für $\mathrm{HPN}_{2}$ sind in Tab. 3 angegeben. Die Positionen der Wasserstoffatome wurden unter Annahme einer $\mathrm{N}-\mathrm{H}$-Bindungslänge von $98 \mathrm{pm}$ berechnet. Die Übereinstimmung zwischen beobachtetem und berechnetem Röntgenbeugungsdiagramm ist in Abb. 2 bzw. Tab. 2 dargestellt.

\section{IR-spektroskopische Untersuchungen}

Das IR-Spektrum (Abb. 3) von kristallinem Phosphor(V)-nitrid-imid $\left(\mathrm{HPN}_{2}\right)$ wurde auf einem FourierTransform-Spektrometer IFS $113 \mathrm{v}$ der Fa. Bruker, Karlsruhe unter Verwendung eines $\mathrm{KBr}$-Preßlings (1 mg $\mathrm{HPN}_{2} / 500 \mathrm{mg} \mathrm{KBr}$ präpariert in einer Glove-Box unter Argon-Schutzgasatmosphäre) aufgenommen. Die Bandenlagen und -zuordnungen sind in Tabelle 5 angegeben. Im Bereich von 400 bis $4000 \mathrm{~cm}^{-1}$ werden 6 Schwingungen beobachtet. Gegenüber früheren IRspektroskopischen Untersuchungen, welche an röntgenamorphen und undefinierten $\mathrm{HPN}_{2}$-Proben durchgeführt wurden $[9,24]$, erhielten wir aufgrund des höheren Ordnungszustandes im kristallinen Festkörper wesentlich geringere Halbwertsbreiten der beobachteten Schwingungsbanden. So gelang es, die jeweils doppelt aufgespaltenen Banden bei etwa 1300 und $950 \mathrm{~cm}^{-1}$ aufzulösen.
Tabelle 5 Schwingungsbanden im IR-Spektrum von kristallinem $\mathrm{HPN}_{2}$

\begin{tabular}{llll}
\hline $3224 \mathrm{~cm}^{-1}$ & $v(\mathrm{~N}-\mathrm{H})$ & $971 \mathrm{~cm}^{-1}$ & $v_{\mathrm{as}}(\mathrm{P}-\mathrm{NH}-\mathrm{P})$ \\
$1330 \mathrm{~cm}^{-1}$ & $v_{\text {as }}(\mathrm{P}=\mathrm{N}-\mathrm{P})$ & $901 \mathrm{~cm}^{-1}$ & \\
$1223 \mathrm{~cm}^{-1}$ & & $531 \mathrm{~cm}^{-1}$ & $\delta(\mathrm{PNP})$ \\
\hline
\end{tabular}

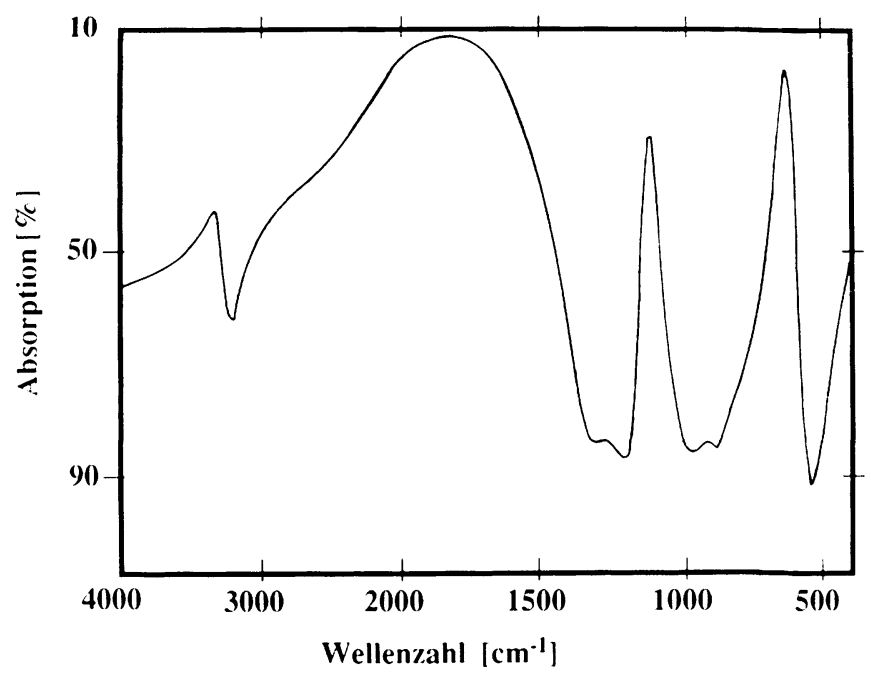

Abb. 3 IR-Spektrum von $\mathrm{HPN}_{2}$

\section{Strukturbeschreibung, Diskussion}

Phosphor(V)-nitrid-imid zeigt im kristallinen Festkörper eine Gerüststruktur ${ }_{\infty}^{3}\left[\mathrm{PN}_{4 / 2}{ }^{-}\right]$aus . eckenverknüpften $\mathrm{PN}_{4}$-Tetraedern, die sich vom isoelektronischen $\beta$-Cristobalit-Typ ableitet (Abb. 4). Gegenüber dem ,,idealen“" C9-Typ (Cristobalit) sind die $\mathrm{PN}_{4}$-Tetraeder jedoch um einen Drehwinkel $\varphi=30,3^{\circ}$ um ihre Drehinversionsachse

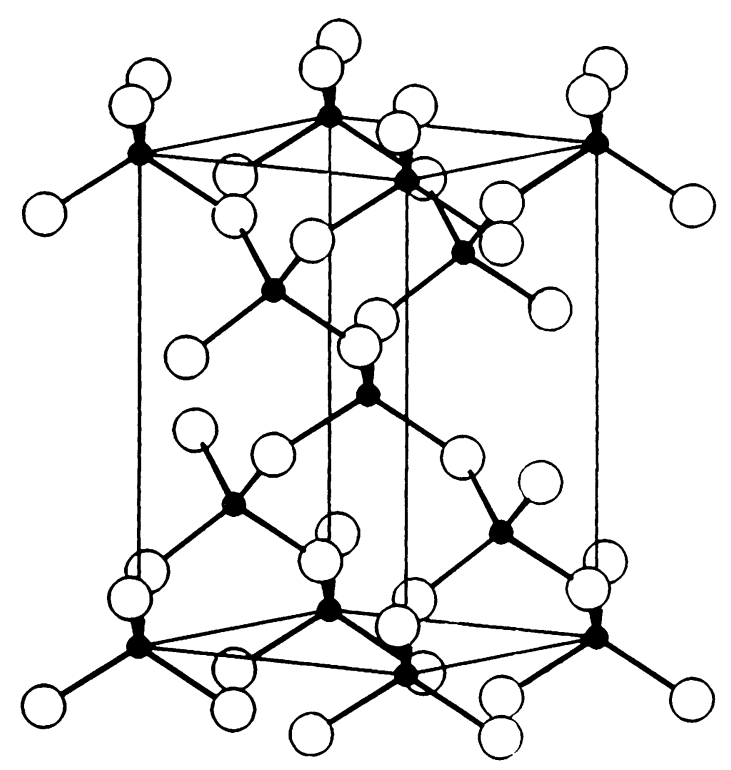

Abb. $4 \beta$-Cristobalit analoge $\mathrm{P}$-N-Teilstruktur in $\mathrm{HPN}_{2}$, Phosphor: schwarz, Stickstoff: weiß 
parallel zur kristallographischen c-Achse verdreht. Benachbarte $\mathrm{PN}_{4}$-Tetraeder sind dabei gegensinnig ausgelenkt. Die beschriebene Verzerrung der Struktur bewirkt einen Übergang vom kubischen (C9-Typ) in das tetragonale Kristallsystem $\left(\mathrm{HPN}_{2}\right)$ gemäß der Basistransformation $(1 / 21 / 20 ; 1 / 2-1 / 20 ; 001)$. Die P-N-Teilstruktur von $\mathrm{HPN}_{2}$ ist damit isotyp der in $\mathrm{LiPN}_{2}$ [2]. Gegenüber der Lithium-Verbindung wird allerdings eine signifikante Verkürzung der $\mathrm{P}-\mathrm{N}$-Bindungslänge von $164,5(7) \mathrm{pm}$ ( $\mathrm{LiPN}_{2}$ [2]) auf $159,9(4) \mathrm{pm}$ in $\mathrm{HPN}_{2}$ gefunden. Damit einher geht eine Verringerung des Drehwinkels von $\varphi=34.2^{\circ}$ in $\mathrm{LiPN}_{2}$ [2] auf $\varphi=30,3^{\circ}$ in $\mathrm{HPN}_{2}$. Gleichzeitig vergrößert sich der $\mathrm{P}-\mathrm{N}-\mathrm{P}$-Bindungswinkel am Stickstoff von $123,6(8)^{\circ}$ in $\mathrm{LiPN}_{2}$ auf $130,1(4)^{\circ}$ in $\mathrm{HPN}_{2}$, während die $\mathrm{N}-\mathrm{P}-\mathrm{N}$-Bindungswinkel in $\mathrm{LiPN}_{2}$ $\left(107,0(5)^{\circ}\right.$ und $114,5(2)^{\circ}$ [2]) nahezu identisch denen in $\mathrm{HPN}_{2}\left(107,5(5)^{\circ}\right.$ und $\left.113,4(5)^{\circ}\right)$ sind.

Die Ursache der beschriebenen strukturellen Variationen zwischen $\mathrm{L}: \mathrm{PN}_{2}$ und $\mathrm{HPN}_{2}$ ist offensichtlich in dem unterschiedlichen strukturchemischen Verhalten von Lithium bzw. Wasserstoff $\mathrm{zL}$ finden. In $\mathrm{LiPN}_{2}$ besetzen die $\mathrm{Li}^{+}$-Ionen tetraedrisch durch Stickstoff koordinierte Lücken der $\mathrm{PN}_{2}{ }^{-}$-Teilstruktur, wobei ene Chalcopyrit-analoge Anordnung resultiert [2]. Demgegenüber sind die Wasserstoffatome in $\mathrm{HPN}_{2}$ weitgehend kovalent an die Hälfte der Stickstoff-Atome des $\mathrm{PN}_{2}{ }^{-}$-Gerüstes gebunden. Die $\mathrm{N}-\mathrm{H}$-Valenzschwingung im IR-Spektrum von $\mathrm{HPN}_{2} \mathrm{t}$-itt bei $3224 \mathrm{~cm}^{-1}$ auf, einem Wert, wie er typischerweise in Inino-Gruppen gefunden wird [18].

Eine endgültige Entscheidung, ob die Wasserstoffatome in $\mathrm{HPV}_{2}$ statistisch über die Hälfte der Stickstoffatome des $\mathrm{PV}_{2}{ }^{-}$-Gerüstes oder geordnet unter Symmetrieerniedrigung verteilt sind, kann aufgrund der vorliegenden röntg€nographischen und spektroskopischen Ergebnisse nicht zetroffen werden. Allerdings bieten sich weder aus der genauen Analyse der Röntgenpulverdaten noch aus den esenfalls angefertigten Eiektronenbeugungsaufnahmen in Mikro-Einkristallen von $\mathrm{HPN}_{2}$ Hinweise auf eine jymmetrieerdniedrigung gegenüber der Kristallstruktur von $\mathrm{LiPN}_{2}$. Zur genauen Ermittlung der Wassersto:f-Positionen in Phosphor(V)-nitrid-imid sind Neutronerbeugungsuntersuchungen an deuterierten $\mathrm{HPN}_{2}-$ Proben geplant.

Phospnor(V)-nitrid-imid ist isoelektronisch zu $\mathrm{SiO}_{2}$ und stellt eine irteressante Modellsubstanz für strukturelle Studien an $\beta$ Cristosalit dar. Erst kürzlich konnten Welberry und Mitarb. überzsugend das Auftreten einer dynamischen Fehlordnung in $\beta$-Crisobalit nachweisen [19-21]. Elektronenbeugungsuntersuchungen im Zusammenhang mit gitterdynamischen Berechnungen führten zu der Annahme gekoppelter Rotationen von $\mathrm{SiO}_{4}-$ Tztraedern in Säulen entlang [110] der kubischen Elementarzele von $\beta$-Cristobalit, wobei die Sauerstoffatome Präzessionstewegungen um die Verbindungslinie benachbarter Siliciumstome ausführen. Als Folge dieser dynamischen Fehlordnung wird beim Röntgenbeugungsexperiment im zeitlichen Mitte die kubische C9-Struktur von $\beta$-Cristobalit $(\mathrm{Fd} \overline{3} \mathrm{~m})$ ge- funden. Im Rahmen einer gitterdynamischen Interpretation wird die beschriebene Präzessionsbewegung durch energetisch tiefliegende und dispersionslose Phononen erklärt [19-21]. Diese führen zu charakteristischen diffusen Ebenen in Elektronenbeugungsaufnahmen von $\mathrm{SiO}_{2}$. Damit war das von Wright und Leadbetter [22] sowie O'Keefe und Hyde [23] geforderte statische Fehlordnungsmodell widerlegt, nach dem die beobachtete kubische Symmetrie von $\beta$-Cristobalit durch Überlagerung von 6 tetragonalen Domänen in der Raumgruppe $I \overline{4} 2 \mathrm{~d}$ vorgetäuscht werde.

In dem $\mathrm{zu} \mathrm{SiO}_{2}$ isosterem Phosphor(V)-nitrid-imid wird nun die im statischen Modell für $\beta$-Cristobalit geforderte tetragonal-verzerrte Ordnungsvariante als Struktur unter Normalbedingungen gefunden, welche beim $\mathrm{SiO}_{2}$ selbst gar nicht auftritt. Bei längerer Untersuchungsdauer im Elektronenmikroskop fanden wir jedoch bei $\mathrm{HPN}_{2}$ identische diffuse Ebenen im Beugungsbild wie im Falle des $\beta$-Cristobalit. Während $\mathrm{HPN}_{2}$ unter Normalbedingungen in der geordneten tetragonalen Struktur kristallisiert, wird offensichtlich durch Aufheizung der Kristallite im Elektronenstrahl eine dem $\beta$-Cristobalit analoge dynamische Fehlordnung thermisch induziert. Dieser Ordnungs-/Unordnungsübergang in $\mathrm{HPN}_{2}$ ereignet sich direkt vor der thermischen Zersetzung der Substanz, so daß bislang ein Nachweis mittels temperaturabhängiger Guinier-Aufnahmen nicht gelang.

Diese Arbeit wurde vom Minister für Wissenschaft und Forschung des Landes Nordrhein-Westfalen, der Deutschen Forschungsgemeinschaft sowie dem Fonds der Chemischen Industrie gefördert. Die Autoren danken Herrn Dr. Frank Krumeich, Arbeitsgemeinschaft Festkörper-Analytik der Universität Bonn, für die Elektronenbeugungsuntersuchungen.

\section{Literatur}

[1] W. Schnick, J. Lücke: J. Solid State Chem. 87 (1990) 101

[2] W. Schnick, J. Lücke: Z. anorg. allg. Chem. 588 (1990) 19

[3] W. Schnick, U. Berger: Veröffentlichung in Vorbereitung

[4] W. Schnick, U. Berger: Angew. Chem. 103 (1991) 857

[5] W. Schnick, J. Lücke: Angew. Chem. 104 (1992) (im Druck)

[6] M. C. Gerhardt: Ann. Chim. Phys. 18 (1846) 188

[7] M. C. Gerhardt: C. R. Acad. Sci. 22 (1846) 858

[8] E. Steger: Angew. Chem. 69 (1957) 145

[9] E. Steger: Chem. Ber. 94 (1961) 266

[10] D. B. Sowerby, L. F. Audrieth: Chem. Ber. 94 (1961) 2670

[11] M. C. Miller, R. A. Shaw: J. Chem. Soc. 1963, 3233

[12] J. M. Sullivan: Inorg. Chem. 15 (1976) 1055

[13] T. N. Miller, A. P. Lodzinya, B. Y. Bondars, A. A. Luzyukevich, A. A. Vitola: Izv. Akad. Nauk SSSR, Neorg. Mater. 15 (1979) 611

[14] G. Boden, G. Sadowski, H. A. Lehmann: Z. Chem. 11 (1971) 114

[15] W. Schnick, J. Lücke: Veröffentlichung in Vorbereitung

[16] O. Buresch, H. G. v. Schnering: Fresenius Z. Anal. Chem. 319 (1984) 418 
[17] STADI/P-Fast Powder Diffractometer System; Fa. Stoe, Darmstadt 1988

[18] J. Weidlein, U.Müller, K. Dehnicke: Schwingungsfrequenzen I, Hauptgruppenelemente; Stuttgart, New York: Thieme Verlag 1981; S. $252 \mathrm{ff}$.

[19] G. L. Hua, T. R. Welberry, R. L. Withers, J. G. Thompson: J. Appl. Crystallogr. 21 (1988) 458

[20] T. R. Welberry, G. L. Hua, R. L. Withers: J. Appl. Crystallogr. 22 (1989) 87

[21] G. L. Hua, T. R. Welberry, R. L. Whiters. J. Phys. Chem. Solids 50 (1989) 207
[22] A. F. Wright, A. J. Leadbetter: Philos. Mag. 31 (1975) 1391

[23] M. O’Keefe, B. G. Hyde: Acta Crystallogr. B 32 (1976) 2923

[24] J. Goubeau, R. Pantzer:Z. anorg. allg. Chem. 390 (1972) 25

Korrespondenzanschrift:

Dr. W. Schnick

Institut für Anorganische Chemie der Universität Gerhard-Domagk-Str. 1

W-5300 Bonn 1, Bundesrepublik Deutschland 